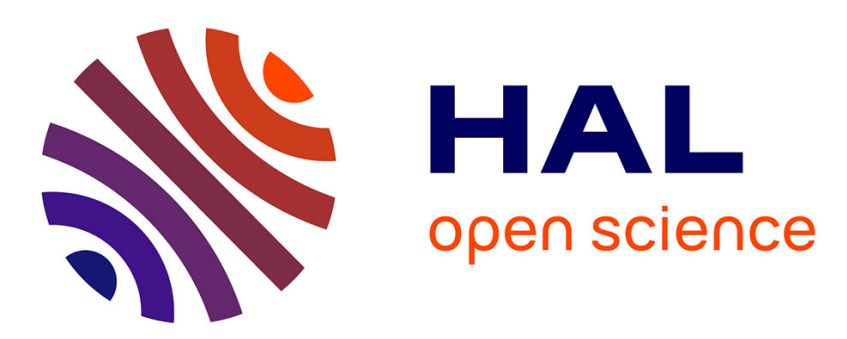

\title{
Schooling properties of an obligate and a facultative fish species
}

\author{
Marc Soria, Pierre Fréon, Pascale Chabanet
}

\section{To cite this version:}

Marc Soria, Pierre Fréon, Pascale Chabanet. Schooling properties of an obligate and a facultative fish species. Journal of Fish Biology, 2007, 71 (5), pp.1257-1269. 10.1111/j.1095-8649.2007.01554.x . ird-00258537

\section{HAL Id: ird-00258537 https://hal.ird.fr/ird-00258537}

Submitted on 4 Mar 2008

HAL is a multi-disciplinary open access archive for the deposit and dissemination of scientific research documents, whether they are published or not. The documents may come from teaching and research institutions in France or abroad, or from public or private research centers.
L'archive ouverte pluridisciplinaire HAL, est destinée au dépôt et à la diffusion de documents scientifiques de niveau recherche, publiés ou non, émanant des établissements d'enseignement et de recherche français ou étrangers, des laboratoires publics ou privés. 


\title{
Schooling properties of an obligate and a facultative
}

\author{
fish species \\ M. SORIA ${ }^{*}$, P. FREON ${ }^{\S}$ and P. CHABANET ${ }^{*}$ \\ *IRD, BP 172, 97492 Sainte-Clotilde cedex, La Réunion, France, ${ }^{\S}$ IRD, Centre de \\ Recherche Halieutique, Avenue Jean Monnet, BP 171, 34203 Sète Cedex, France and \\ ${ }^{\ddagger} I R D, B P$ A5, 98848 Nouméa cedex, Nouvelle-Calédonie, France
}

Schooling fish species are conventionally subdivided into obligate and facultative species, depending on the proportion of the time spent in structured schools, but little is known about the factors affecting the group structure in the absence of external structuring stimuli such as food sources, risk of predators or water flow. Changes in attraction and repulsion indicators, depending on the species and the group size were explored under controlled conditions. Two species, displaying different schooling behaviours in the wild were observed: the bigeye scad Selar crumenophthalmus (Bloch) and the barred flagtail Kuhlia mugil (Forster). In the bigeye scad, the Polarity and Speed were high and stable, and the Nearest Neighbour Distance $(N N D)$ decreased when the group size increased. In contrast, for the barred flagtail, Polarity and Speed decreased according to the group size, inducing a loss of cohesion and leading to a disorganized school. The $N N D$ mean was stable whatever the group size and relatively high. This experiment indicated that the ability to polarize is first a species specific trait, rather than a property emerging from the group and led by the circumstances.

Key words: Social interactions, Schooling behaviour, Polarity, Pelagic fish

Running headline: Schooling properties of two fish species

*Author to whom correspondence should be addressed. Tel.: +262 2622993 17; fax: +262 2622848 79; email: soria@la-reunion.ird.fr 


\section{INTRODUCTION}

Pelagic fish schools provide a good example of a social structure that enables individuals to increase their efficiency when foraging and avoiding predators by performing complex and synchronous movements that are beneficial to each individual school member (Pitcher \& Parrish, 1993; Parrish \& Edelstein-Keshet, 1999; Krause et al., 2000). Previous observations and analyses of swimming fish in aquaria (Breder, 1951; Shaw \& Tucker, 1965; Hemmings, 1966; Shaw, 1970; Radakov, 1973; Aoki, 1980) defined sense organs and forces between fish implied in the schooling behaviour. Several of these experiments suggested that fish, like other social animals, had an "exclusive sphere" from which conspecifics were expelled. Moreover, Shaw \& Tucker (1965) defined the optomotor reaction as the ability of fishes to synchronize their motions and to adjust their speeds to those of their neighbours. This reaction is one of the most important orientation mechanisms that enable fish to maintain their position and their orientation within a school (reviewed by Warburton, 1997). Consequently, one of the general rules of social behaviour is that schooling fish attempt to maintain a minimum distance between conspecifics (reviewed by Krause \& Ruxton, 2002). Polarity measures the tendency of individuals to align with one another and to synchronize their motions. Fish schools under natural condition display a wide range of polarity values over time, from completely non-polarized to perfectly aligned (Shaw, 
1978). This characteristic led Breder (1959) to distinguish between obligate schooling fishes which polarized constantly and facultative schooling fishes which only polarized occasionally. This classification was used by Pitcher (1983) to distinguish a shoal (unstructured aggregation) from a school (social structure enabling quick information to be transferred to conspecifics). However, under natural conditions, several external factors such as food source, risk of predators or water flow may have an impact on the cohesion of fish congregation. Differences in hunger (Morgan, 1988), fear (Rehnberg \& Smith, 1988), fish size (Parrish \& Turchin, 1997), species composition (Allan, 1986) or in group size (Fitzsimmons \& Warburton, 1992) may promote individual differences in behaviour that generate variation in the internal organisation of fish schools and ultimately affect the structure and stability of groups. A "real fish school", defining a fully polarized fish school under natural conditions, could be a temporary and spontaneous response of a shoal to a disturbance or predator. Therefore, until the constraints on fish and the processes enabling them to adjust their positions and synchronize their motions are not fully understood, it seems difficult to deduce from observations in the wild which species are obligate schooling fishes or facultative schooling fishes (Viscido et al., 2004; Wu \& David, 2002).

The question then becomes: what governs attraction and repulsion between individuals in the absence of any external structuring stimuli (i.e. when the sources of attraction/repulsion are the fish themselves)? To address this issue, 
behavioural interactions among fishes were examined under controlled conditions depending on different group sizes. Polarization is important in minimizing collision between individuals and in allowing the group to transfer information (Radakov, 1973). Therefore, two gregarious species were compared under controlled conditions to check the internal factors influencing this collective behaviour.

\section{MATERIALS AND METHODS}

The first studied species was a Carangidae, the bigeye scad Selar crumenophthalmus (Bloch). This small circumtropical pelagic fish is an a priori obligate schooling fish, travelling in compact groups of hundreds of thousands of fish. In the coastal area around Reunion Island (south-western Indian Ocean), their main natural predators are the giant trevally Caranx ignobilis (Forsskål) and the Scombridae Kawakawa Euthynnus affinis (Cantor). The second species studied is a Kuhlidae, the barred flagtail Kuhlia mugil (Forster). This small pelagic coastal tropical fish is an a priori facultative schooling fish living in shoals along the outer slope of the coral reefs.

Barred flagtail were caught in March 2001, bigeye scad in March 2002. Fishes were caught using a small sliding net of $3.5 \mathrm{~m}$ in length, $6 \mathrm{~m}$ in height and a mesh size of 10 $\mathrm{mm}$. Fishes were gently guided into plastic bags to avoid wounds during extraction from 
the net, and transferred into 601 buckets under hyper-oxygenation and a soft anaesthesia ( $0.1 \mathrm{ml} \mathrm{l}^{-1}$ of clove oil, Durville \& Collet, 2001). For experiments on both species, 80 to 100 fishes were finally conveyed to the marine station and housed in a holding tank of $4 \mathrm{~m}$ diameter and $1.2 \mathrm{~m}$ high. A preventive treatment with methylene blue $\left(0.2 \mathrm{mg} \mathrm{l}^{-1}\right)$ and formalin (20 $\left.\mathrm{mg} \mathrm{l}^{-1}\right)$ was applied in order to avoid parasitism and bacterial or fungal infections. Fishes were fed daily ad libitum with a mixture of aquaria flake-food and pieces of fish flesh. Fishes were considered acclimatized when all of them fed on the aquaria flake-food. This weaning period lasted about 15 days. Fork length of studied fishes varied from $19-22 \mathrm{~cm}$ for the bigeye scad, and from $22-25 \mathrm{~cm}$ for the barred flagtail.

The experiments were performed over six months, in both April to June 2001 and April to June 2002 at the Sea Turtle Survey and Discovery Centre of Reunion Island. A circular tank of $4 \mathrm{~m}$ diameter and $1.2 \mathrm{~m}$ high similar to the holding tank was used. Opaque curtains were placed around and above the tank to obtain diffuse lighting and to reduce external disturbances from the environment. The tank was supplied with a continuous flow of seawater (Domenici et al., 2000). Since currents may influence fish behaviour, the seawater inlet pipe was placed vertically and the water flow was stopped throughout the observation periods. A digital video camera (Sony model CDR-TRV 900E) was fixed at five meters above the tank and tilted at $45^{\circ}$ to observe the totality of the tank. The remotely operated video camera was fitted with a polarizing filter and a wide-angle lens. $80 \%$ of the trials were performed in the morning to avoid possible conditions of strong wind that may disturb the fish, and sunshine that may render light inside the tank unsuitable for video recording. Prior to each trial, the fish were deprived of food for 12 
hours to standardize the hunger level and were transferred to the experimental tank and acclimatized to their new environment for a period of 20 minutes.

Seventy (70) barred flagtail and 85 bigeye scad were used. For each species, three group sizes were examined: two, four and eight individuals for the bigeye scad and two, five and 10 individuals for the barred flagtail. Five replicates per species and group size were performed. For each replicate, fish behaviour was recorded during five minutes. Three different typical and stable patterns of aggregation (line, column and dense school) were easily but empirically identified during the experiment on four and eight bigeye scad and were post-processed accordingly. Their frequencies were estimated for each replicate by measuring the duration of each pattern over the observation period.

Preliminary trials indicated that, occasionally, one fish might display a fleeting alarm reaction. Because this reaction was undesirable for the purpose of this study, only two minutes without disturbances were retained out of the five recorded. The data processing consisted in sampling one image per second out of the 24 images recorded by the video camera. Then, the coordinates of each fish were recorded using software specially developed for this experiment. In order to convert pixels into distance units and to take into account the parallax error due to the tilt of the video camera and the distortion of the image related to the wide-angle lens, an empirical method was applied. It consisted in recording evenly spaced reference points (every $0.50 \mathrm{~m}$ ) marked out on the bottom of the tank before the beginning of the experiment. The number of pixels observed between these markers on the image was used to calculate the exact coordinates. Two zones were defined 
in the tank: a central zone, where the fish were not affected by the wall, and a peripheral zone, where the fish underwent the edge effect. These zones were defined by measuring the variations of the mean angle between the direction of solitary barred flagtail fish (alone in the tank) and the tangent of the wall versus the distance to the wall. The relationship between these two variables displayed a breaking point at $0.3 \mathrm{~m}$ from the wall (not shown). In order to test the homogeneity of the spatial distribution, the observed proportion of fish between the peripheral $\left(3.5 \mathrm{~m}^{2}\right)$ and the central zones $\left(9 \mathrm{~m}^{2}\right)$ was compared to the expected proportion under the null hypothesis of a random distribution using a chi square test $\left(\chi^{2}\right)$. Finally, the third (vertical) dimension of schools was neglected since the shallow water of the tank $(1.1 \mathrm{~m})$ precluded serious errors in the horizontal measures and the fish tended to use only the middle part of the water column.

Four variables were analyzed: the Nearest Neighbour Distance (NND, in $\mathrm{m})$, the Speed $\left(V\right.$, in $\left.\mathrm{m} \mathrm{s}^{-1}\right)$, the Expanse ( $\varepsilon$, dimensionless) and the Polarity ( $\Phi$, dimensionless). The $N N D$ for each fish was found by computing the minimum distance from one fish to all the individuals in the area. $V$ (in $\mathrm{m} \mathrm{s}^{-1}$ ) was estimated by the linear distance between the positions of the heads of the fish in two successive images (of one-second interval). As far as $V$ and $N N D$ were concerned, for each interval of one second, one value among the values calculated for each individual (for $V$ ) or for pairwise individuals (for $N N D$ ) was randomly selected. Expanse is the mean quadratic distance from each individual to the centre of the group. It gave an accurate estimation of the fish dispersion (Huth \& Wissel, 1992). Expanse ( $(\varepsilon)$ is expressed as:

$$
\varepsilon=\sqrt{\sum_{i=1}^{h}(|X i|-|C|)^{2} h^{-1}}
$$


where $h$ is the number of fish in a group, $X i$ the position vector of individual $i$ and $C$ the position vector of the group.

The group Polarity $\left(\Phi^{*}\right)$ was estimated by the mean of $\alpha i$, the vector angle deviation between the group and the individual courses (Huth \& Wissel, 1992). The group Polarity $\left(\Phi^{*}\right)$ is expressed by the following equation:

$$
\Phi *=\frac{1}{h} \sum_{i=1}^{h} \alpha i(2)
$$

Because this metric is counter-intuitive, a non-dimensionalized form of polarity was used in order to obtain values ranging from 0 (non-polarized) to 1 (perfectly aligned), as in Viscido et al. (2004). This new Polarity $\Phi$ is defined as:

$$
\Phi=\left(90-\Phi^{*}\right) 90^{-1} \text { (3) }
$$

Let $\boldsymbol{V} i$ represent the unit vector for individual fish $i, U$ the unit vector of the group centre (scaled to preserve direction) and $\beta_{i}$ the transformed turning angle $\theta_{i}$ as $\beta_{i}=90^{\circ}-\theta_{i}$ ( $\theta_{i}$ being the difference of course of the fish between two time steps). The vector angle deviation $\alpha_{i}$, between group and each individual course is the inverse cosine of the scalar product $\boldsymbol{V i} U$ :

$\alpha_{i}=\arccos \left(v_{i} U|U|^{-1}\right)(4)$

where:

$$
\begin{aligned}
& v_{i} U=\cos \left(\beta_{\mathrm{i}}\right)\left(\sum_{i=1}^{h} \cos \left(\beta_{\mathrm{i}}\right)\right)+\sin \left(\beta_{\mathrm{i}}\right)\left(\sum_{i=1}^{h} \sin \left(\beta_{\mathrm{i}}\right)\right) \\
& |U|=\sqrt{\left(\sum_{i=1}^{h} \cos \left(\beta_{\mathrm{i}}\right)\right)^{2}+\left(\sum_{i=1}^{h} \sin \left(\beta_{\mathrm{i}}\right)\right)^{2}}
\end{aligned}
$$


If the positions were collected too frequently, the data set might be oversampled due to redundant data points. Therefore, to address the issue of the independence of consecutive observations, data were aggregated over an appropriate interval resulting in independent observations. The optimal interval was found by performing autocorrelation and spectral (Fourier) analyses in order to detect positive serial correlations between discrete values and to assess the periodicity in the time series.

Firstly, effect of replicate was tested solely by performing a series of one-way analyses of variance (ANOVA) and secondly by comparing the effect of replicate to other factors (Group Size and Species) on the four variables using a multifactor analysis of variance (Multifactor ANOVA). Although Multifactor ANOVA is robust to non-gaussian distribution, for bigeye scad data set, $N N D$ right-skewed data were log-transformed and a Box-Cox transformation (Box \& Cox, 1964) was applied to $\Phi$ left-skewed data with an optimal $\lambda$ value of 8 that removed the skewness: $f(\Phi)=\left(\Phi^{\lambda}-1\right) \lambda^{-1}$. Other variables did not require transformation. However, bigeye scad's Speed had one outlier observation (out of 435), which had to be removed.

In order to detect possible non-linear relationships, General Additive Models (GAMs) were applied using loess smoothing with span value adjusted to optimally remove obvious trends in the residuals. For linear relationships, the correlation coefficient matrices for bivariate relationships were calculated and the relative effects of group size and grouplevel characteristics on $\Phi$ were quantified using a General Linear Model (GLM). 


\section{ETHICAL NOTE}

Several procedures were used to optimise the welfare of fish. The fishing operation was carried out without fish being directly handled or emerged in order to avoid injuries and losses of scales that are often the first cause of mortality. The mortality rate observed during transfer from the fishing boat to the experimental station was $4 \%$. The continuous seawater flow in both tanks enabled us to maintain a suitable temperature and oxygen content $\left(\mathrm{T}^{\circ}=28.3 \pm 0.5^{\circ} \mathrm{C} ; \mathrm{O}^{2}=5.90\right.$ $\pm 0.02 \mathrm{mg} \mathrm{l}^{-1}$ ). The maximum density inside the holding tank was less than three fish $\mathrm{m}^{-3}$. During the experiments, low mortality occurred (five fishes in 2001 and five fishes in 2002). These losses resulted from skin wounds and occurred when the fishes rasped against the net during their capture. Three months later, at the end of the experiment, the fish were released at their site of capture. Thus, one can be confident that the experiments had no significant effect on the population and did not cause undue stress to the subjects.

\section{RESULTS}

Autocorrelation and Fourier analyses (results not shown) performed on each variable for each factor indicated that a 4-second time-average was appropriate to 
avoid biases due to autocorrelated data or periodicity. This time average led us to analyse 29 image samples by replicate. The effect of repeated measurements was significant on most of the variables (ANOVA, $\mathrm{n}=145, \mathrm{P}<0.001$ ), except for the barred flagtail's $N N D$ and the bigeye scad's Polarity and Expanse for small and medium groups. However, the Multifactor ANOVAs performed with Species, Group Size and replicate as factors on each variable have shown that the percentage of variance due to replicate was lower than $5 \%$ of the total variance (results not shown).

Comparison between the ratio of the number of fish observed in central zones to the number in the peripheral zones and the expected ratio that would result from homogeneous spatial distribution have shown contrasted results between the two species. For the bigeye scad, density was higher than expected in the peripheral zone and lower in the central zone for the small and the medium group sizes $\left(\chi^{2}, n\right.$ $=1200$, d.f. $=1, \mathrm{P}<0.05 ; \chi^{2}, \mathrm{n}=2400$, d.f. $=1, \mathrm{P}<0.01$ respectively), whereas fish densities observed in both zones for the large group size were not statistically different $\left(\chi^{2}, n=4800\right.$, d.f. $\left.=1, P=0.51\right)$. Therefore, analyses indicated a definite thigmotaxis, i.e. a trend to look for contacts and to maintain its body parallel to the tank wall, for bigeye scad, mainly for small and medium groups. In contrast, for the barred flagtail density was always higher in the central zone than in the peripheral zone whatever the Group Size $\left(\chi^{2}, \mathrm{n}=1200\right.$, d.f. $=1, \mathrm{P}<0.001 ; \chi^{2}, \mathrm{n}=$ 
3000, d.f. $=1, \mathrm{P}<0.001 ; \chi^{2}, \mathrm{n}=6000$, d.f. $=1, \mathrm{P}<0.001$ for small, medium and large group sizes respectively).

Means of Polarity, Expanse, Speed, and NND for both species and each group size are summarized in Table I. Since GAMs did not show non-linear relationships between the main group-level characteristics, the product-moment correlation coefficients were analysed and the influence of factors and other variables on Polarity were tested using a GLM.

For bigeye scad, the mean Polarity was high, regardless of the group size (0.81 to 0.89 ; Table I). The mean Expanse was small and more or less stable (0.22 to 0.28 ) whatever the group size. These differences (from $5 \%$ to $22 \%$ ) were sometimes statistically significant (Table I) but the relationship between Expanse and Group Size was weak $(\mathrm{r}=-0.24, \mathrm{n}=145, \mathrm{P}<0.001$, Table II). $N N D$ decreased dramatically and significantly with increasing group size $(\mathrm{r}=-0.84, \mathrm{n}=$ 145, $\mathrm{P}<0.001$, Fig. 1), indicating that fish adjusted their distances according to the number of individuals in the group. Expanse and $N N D$ were positively correlated $(r=0.75, n=435, P<0.001$, Table II). This result suggests that, for this species and for the tested Group Size range, Expanse was first a group-level expression of $N N D(\mathrm{r}=0.97, \mathrm{n}=145, \mathrm{P}<0.001 ; \mathrm{r}=0.95, \mathrm{n}=145, \mathrm{P}<0.001 ; \mathrm{r}=$ 
$0.55, \mathrm{n}=145, \mathrm{P}<0.001$ for small, medium and large groups respectively), but not scaled to Group Size. Mean Speed decreased significantly with increasing Group Size $(\mathrm{r}=-0.29, \mathrm{n}=435, \mathrm{P}<0.001$, Tables I and II). Polarity was positively correlated with Speed $(\mathrm{r}=0.56, \mathrm{n}=435, \mathrm{P}<0.001$, Table II). Therefore, increasing group size led to an individual speed decrease associated with a decrease in Polarity. The GLM showed an expected strong and significant effect of Speed on Polarity, a weak but significant effect of Group Size, and no significant effect of $N N D$ and Expanse, indicating that schools always tended to display perfect alignment, regardless of these last two variables (Table III).

Detailed observations of medium (four individuals) and large (eight individuals) group sizes have shown that bigeye scad displayed three different and specific spatial patterns. The fishes could follow each other forming a column, swam abreast forming a line or got into small but dense schools (Fig. 2). Analyses have shown that the three patterns were observed in equivalent proportions in the group of four individuals $(32.7 \pm 9.3 \%, 33.8 \pm 7.8 \%, 33.5 \pm 14.2 \%$ for line, column and dense school patterns respectively; $\chi^{2}, \mathrm{n}=584$, d.f. $=2, \mathrm{P}>0.9$ ) whereas the group of eight individuals displayed on average the third pattern more often than the other ones $(14.4 \pm 6.3 \%, 10.5 \pm 5.7 \%, 75.0 \pm 9.8 \%$ for line, column and dense school patterns respectively; $\chi^{2}, \mathrm{n}=595$, d.f. $\left.=2, \mathrm{P}<0.001\right)$. In large Group Size, schooling fish that turned back altogether most often kept the school 
structure. This behaviour maintained a cohesive pattern and induced small $N N D$ throughout the observations. Most strikingly, the fish swam along the wall until they reached the opposite part of the tank, turned back and started again in the opposite direction after they had covered more or less half the perimeter of the tank.

For barred flagtail, Polarity and Expanse were negatively correlated $(\mathrm{r}=$ $0.70, \mathrm{n}=435, \mathrm{P}<0.001)$ and both variables were significantly affected by the size of the group (Tables I and II). Polarity decreased with Group Size $(\mathrm{r}=-0.73, \mathrm{n}=$ 435, $\mathrm{P}<0.001)$ whereas Expanse increased $(\mathrm{r}=0.80, \mathrm{n}=435, \mathrm{P}<0.001)$. In other words, increasing group size destroyed the alignment and led to an expansion of the group. The correlation between Expanse and $N N D$ was not significant $(\mathrm{r}=$ $0.09, \mathrm{n}=435, \mathrm{P}=0.055)$. The correlation between Group Size and $N N D$ was significant but low $(\mathrm{r}=-0.26, \mathrm{n}=435, \mathrm{P}<0.001)$, indicating that distance between neighbours is slightly dependent on the group size (Fig. 1). However, the ANOVA has shown that $N N D$ mean was significantly lower for the large group $(0.33 \mathrm{~m})$ compared to the small and medium group sizes $(0.41$ and $0.42 \mathrm{~m}$ respectively, Table I). In other words, the spatial occupation and the heterogeneity of spatial arrangement of fishes increased with group size. Speed variability of barred flagtail was similar to that of bigeye scad. Speed decreased significantly with increasing Group Size $(\mathrm{r}=-0.55, \mathrm{n}=435, \mathrm{P}<0.001$, Table I and II). Polarity 
was positively correlated with Speed $(\mathrm{r}=0.64, \mathrm{n}=435, \mathrm{P}<0.001$, Table II). However, mean Speed for barred flagtail was significantly lower than for bigeye scad, within each group size (repeated measures ANOVA: $\mathrm{n}\left({ }_{\text {barred flagtail }}\right)=145$ and $\mathrm{n}($ bigeye scad $)=145$, d.f. $=4, \mathrm{P}<0.001$ for the three group sizes $)$. Therefore, increasing group size led to an individual speed decrease associated with a decrease in Polarity. Moreover, the standard errors of mean Speed for the three group sizes were high but similar (Table I), indicating that the loss of cohesion with increasing group size was not due to a greater difference between individual motions.

\section{DISCUSSION}

Cohesion of fish schools is essential to survive in the risky and open pelagic domain. The analysis of the four variables (NND, Speed, Expanse and Polarity) linked to the attraction and repulsion forces governing this cohesion for two pelagic species observed in three different group sizes and in the absence of external structuring stimuli have shown contrasted results in terms of the arrangement of individuals inside the school. For the bigeye scad $S$. crumenophthalmus, the fishes were found parallel to each other, with Polarity values always greater than 0.81 and displaying little variation when Group Size 
varied within the tested range of 2 to 8 individuals. Polarity was unrelated to the dispersion index (Expanse) and it was weakly variable depending on Speed. This means that most of the time, fish were perfectly polarized and sustained only three stable and regular patterns: line, column and dense school. Mean $N N D$ decreased dramatically with increasing group size, indicating that the bigger the group, the higher the density. These results suggest that the bigeye scad maintained group cohesion whatever the group size and formed closed ranks when group size increased. In other words, all individuals were mutually influential neighbours to each other and individuals adjusted their speeds and maintained closest contact between them. Most strikingly, high polarization and decreasing $N N D$ with increasing Group Size appeared in the absence of external factors such as food source, risk of predators or water flow (Hoare et al., 2004). These observations confirm that the bigeye scad forms real fish schools and can be classified as an obligatory gregarious species. This interpretation is reinforced by the observed positive thigmotaxis, particularly for small group sizes, that may be due to a deficiency of conspecifics and then could be attributed to a social taxis, i.e. the search for conspecifics (Grünbaum, 1997). The possible presence of informed school leader(s) (Reebs, 2000) falls beyond the scope of this study. Although this presence cannot be totally excluded, especially when fish move in columns, this factor is not essential here, due to the lack of external stimuli. 
Barred flagtail groups were less structured and moved slower than bigeye scad. Only pairs of barred flagtail (small Group Size) maintained a high cohesion $(\Phi=0.79$ on average $)$ and a medium Speed $\left(0.36 \mathrm{~m} \mathrm{~s}^{-1}\right)$, but both values decreased dramatically with increasing Group Size, inducing both a loss of cohesion and a disorganization inside the shoal. This result was emphasized by a high dispersion index for the medium and large group sizes. The mean $N N D$ was relatively stable but high with $0.40 \mathrm{~m}$ on average ( 2 body lengths) which is twice as high as the mean $N N D$ most often quoted in the literature for small schooling pelagic fish (Pitcher \& Parrish, 1993; Fréon \& Misund, 1999). By pair, a given individual has elected one conspecific by maintaining almost permanently the same swimming direction but in a larger group size, fish was weakly stimulated by the other members. This result is in agreement with Romey (1996) who showed that inter-individual forces alone are enough to characterize the trajectories of simulated groups. Therefore, for this species, social interactions can be considered as tenuous, mainly accomplished via pairwise interactions, and not mainly due to the mutual attraction of individuals. In this way, the barred flagtail can be listed as the facultative gregarious species. 
The main difference between the two species was their ability to form polarized group. Since the observations were conducted in the absence of external factors, it was assumed that the observed movement properties of individuals were correlated to species, and deduced that the ability to polarize is first a species specific trait. For obligate schooling fishes like bigeye scad, conspecifics should obtain this pattern by matching the velocity with their neighbours and by decreasing $N N D$, leading to a densification of the group that nonetheless retains a high velocity. This pattern regulating the movement of individuals inside the group should result from natural selection at the individual level rather than from a collective and emergent outcome, not directly controlled by any member. In other words, the degree of social motivation (related to species) can be linked to the general attraction/repulsion rules between conspecifics. Moreover, the tendency of individuals to align with one another within the polarized group allows efficient information transfer and fast reaction to predators (e.g. Gerlotto et al., 2006). Therefore, from an ecological and evolutionary point of view, polarity is a species specific trait under low influence of external factors.

The results on barred flagtail, classified as a facultative schooling fish in this study, were similar to those obtained by Parrish \& Turchin (1997) on the juvenile blacksmith (Chromis punctipinnis (Cooper), family Pomacentridae) 
and by Viscido et al. $(2004,2005)$ on the giant danios (Danio aequipinnatus (McClelland), family Cyprinidae). However, the experimental results on bigeye scad presented here are different from those obtained on simulated danio by Viscido et al. (2004), in two ways. First, Polarity was less group sizedependent in the present study than in previous ones: no change in Polarity was observed from two to four individuals, and only a decrease of $9 \%$ from four to eight individuals was found, compared to a decrease of $28 \%$ for danios in Viscido et al. (2004). Second, the observed distance between fishes varied in the present study according to Group Size showing that bigeye scads did not have a preferred $N N D$ linked to their group size. NND decreased from 0.54 to $0.12 \mathrm{~m}$ when Group Size changed from two to eight individuals, while a nonsignificant increase was observed for danios (from 0.12 to $0.17 \mathrm{~m}$ ) by Viscido et al. (2004). In the same way, if simulated danios formed large and persistent groups, those groups were never very highly polarized (Viscido et al., 2005). Therefore, the results of the present work imply that like the barred flagtail, the danio is a facultative schooling fish.

Several authors indicated that fish schools observed in situ displayed heterogeneous and variable structures at large scale (Fréon \& Misund, 1999; Gerlotto \& Paramo, 2003). Fréon et al. (1992) described the global internal structure of a whole fish school in the wild (made up of hundreds of thousands 
of individuals). On a large scale, a fish school was a very heterogeneous 3D structure, with numerous empty sub-volumes (named "vacuoles"), and distant groups of fish displayed different alignments and behaviour. Tensions induced splitting and stretching behaviour and generated a more heterogeneous structure. This was probably due to a higher variability of individual characteristics in the wild and the effect of external factors like a patchy distribution of prey (Pitcher \& Parrish, 1993; Parrish \& Turchin, 1997). In the same way, the behaviour of facultative schooling fishes in natural conditions could lead individuals to align to each other within a parallel group. Several reasons have been advanced to explain these natural patterns, such as the number of influential neighbours (Warburton \& Lazarus, 1991; Huth \& Wissel, 1992) or external conditions (risk of predators, food availability, dissolved oxygen, hydrodynamism) which have an effect on fish behaviour by favouring or forcing alignment (Moss \& McFarland, 1970; Abrahams \& Colgan, 1985; Sogard \& Olla, 1997; Hoare et al., 2004). Only obligate schooling fishes should be able to form a real fish school, but in the wild, an aggregation is a dynamic entity exhibiting a range of structures and spacing according to circumstances (Nottestad \& Axelson, 1999; Viscido et al., 2005). Therefore, more observational data in natural conditions are needed to better understand how fish groups function and how social behaviours evolve in the wild. 
The comparison of this experimental work with the modelling approach allows to have a better understanding of the differences between facultative and obligate schooling fish species. Two different modelling approaches have already been used: a "threshold tendency" approach, used by Viscido et al. (2004, 2005), postulating a fixed repulsion zone around each individual in a group (Aoki, 1982; Huth \& Wissel, 1992; Couzin et al., 2002) and a "continuous tendency attraction-repulsion" model, where attraction increases and repulsion decreases when inter-individual distance increases (Warburton \& Lazarus, 1991; Warburton, 1997). Since bigeye scads seem to be able to adjust their NND according to the number of conspecifics by closing ranks, the increased cohesion between fish with increase in the individual number might be viewed as the decrease of the width of the repulsion zone surrounding each individual. Therefore, the results of this study support the "continuous tendency" model for bigeye scad and the "threshold tendency" approach for barred flagtail. The senses of the facultative schooling fishes like barred flagtail may not be efficient enough to operate an adjustement of the width of the attraction-repulsion and orientation zones, while this should be the case for the obligate schooling fishes like bigeye scad. Therefore, the rules for each model could account for the difference between schooling types described previously by Breder (1959). 
The authors are grateful to the team of the Sea Turtle Survey and Discovery Centre of Reunion Island and especially its Manager S. Cissione. J. Gautrais, G. Theraulaz, G. Lemartin, F. Beudard, A. Dimakopoulos, K. Addi and S. Hoareau are thanked for their assistance during data acquisition and data processing and for helpful comments. Comments and constructive criticisms from anonymous referees are acknowledged. This work is a contribution of the research units R109 THETIS and R097 ECO-UP of the IRD.

\section{References}

Abrahams, M. V. \& Colgan, P. W. (1985). Risk of predation, hydrodynamic efficiency and their influence on school structure. Environmental Biology of Fishes 13, 195202.

Allan, J. R. (1986). The influence of species composition on behaviour in mixed-species cyprinid shoals. Journal of Fish Biology 29, 97-106. doi: 10.1111/j.10958649.1986.tb05002.x

Aoki, I. (1980). An analysis of the schooling behavior of fish: internal organization and communication process. Bulletin of the Oceanic Research Institute of the University of Tokyo 12, 1-65.

Aoki, I. (1982). A simulation study on the schooling mechanism in fish. Bulletin of the Japanese Society of Scientific Fisheries 48, 1081-1088.

Box, G. E. P. \& Cox, D. R. (1964). An analysis of Transformations. Journal of the Royal Statistical Society 26, 211-243. 
Breder, C. M. Jr. (1951). Studies one the structure of the fish school. Bulletin of the American Museum of Natural History 98, 1-28.

Breder, C. M. Jr. (1959). Studies one social groupings in fishes. Bulletin of the American Museum of Natural History 117, 393-482.

Couzin, I. D., Krause, J., James, R., Ruxton, G. D. \& Franks, N. R. (2002). Collective memory and spatial sorting in animal groups. Journal of Theoretical Biology 218, $1-11$.

Domenici, P., Steffensen, J. F. \& Batty, R. S. (2000). The effect of progressive hypoxia on swimming activity and schooling in Atlantic herring. Journal of Fish Biology 57, 1526-1538. doi: 10.1111/j.1095-8649.2005.00884.x

Durville, P. \& Collet, A. (2001). Clove oil as an anaesthetic with juvenile tropical marine fishes. Live Reef Fish Information Bulletin 9, 17-19.

Fitzsimmons, S. D. \& Warburton, K. (1992). Fish movement behaviour: variability within and between groups. Behavioural Processes 26, 211-216.

Fréon, P. \& Misund, O. A. (1999). Dynamics of pelagic fish distribution and behaviour: effects one fisheries and stock assessment. London: Blackwell Science. Fishing News Books.

Fréon, P., Gerlotto, F. \& Soria, M. (1992). Changes in school structure according to external stimuli: description and influence on acoustic assessment. Fisheries Research 15, 45-66.

Gerlotto, F. \& Paramo, J. (2003). The three-dimensional morphology and internal structure of clupeid schools as observed using vertical scanning multibeam sonar. Aquatic Living Resources 16, 113-122. 
Gerlotto, F., Bertrand, S., Bez, N. \& Gutierrez, M. (2006). Waves of agitation inside anchovy schools observed with multibeam sonar: a way to transmit information in response to predation. ICES Journal of Marine Science 63, 1405-1417.

Grünbaum, D. (1997). Schooling as strategy for taxis in a noisy environment. In Animal groups in three dimension (Parrish, J.K. \& Hamner W.M., eds.), pp. 257-281. Cambridge: Cambridge University Press.

Hemmings, D. C. (1966). Olfaction and vision in fish schooling. Journal of Experimental Biology 45, 449-464.

Hoare, D. J., Couzin, I. D., Godin, J.-G. J. \& Krause, J. (2004). Context-dependent group size choice in fish. Animal Behaviour 67, 155-164.

Huth, A. \& Wissel, C. (1992). The simulation of the movement of fish schools. Journal of Theoretical Biology 156, 365-385.

Krause, J. \& Ruxton, G. D. (2002). Living in groups. Oxford: Oxford University Press.

Krause, J., Hoare, D. J., Croft, D., Lawrence, J., Ward, A., Ruxton, G. D., Godin, J-G. J. \& James, R. (2000). Fish shoal composition: mechanisms and constraints. Proceedings of the Royal Society of London 267, 2011-2017.

Morgan, M. J. (1988). The effect of hunger, shoal size and the presence of a predator on shoal cohesiveness in bluntnose minnows, Pimephales notatus Rafinesque. Journal of Fish Biology 32, 963-971. doi: 10.1111/j.1095-8649.1988.tb05439.x

Moss, S. A. \& McFarland, W. N. (1970). The influence of dissolved oxygen and carbon dioxide on fish schooling behaviour. Marine Biology 5, 100-107.

Nottestad, L. \& Axelson, B.E. (1999). Herring schooling manoeuvres in response to killer whale attacks. Canadian Journal of Zoology 77, 1540-1546. 
Parrish, J. K. \& Turchin, P. (1997). Individual decisions, traffic rules, and emergent pattern in schooling fish. In Animal groups in three dimensions (Parrish, J.K. \& Hamner W.M., eds.), pp. 126-142. Cambridge: Cambridge University Press.

Parrish, J. K. \& Edelstein-Keshet, L. (1999). Complexity, pattern, and evolutionary trade-offs in animal aggregation. Science 284, 99-101.

Pitcher, T. J. (1983). Heuristic definitions of shoaling behaviour. Animal Behaviour 31, 611-613.

Pitcher, T. J. \& Parrish, J. K. (1993). Functions of shoaling behaviour in teleosts. In: The Behaviour of Teleost Fishes (Pitcher T.J. ed.), $2^{\text {nd }}$ ed., pp. 363-427. London: Chapman \& Hall.

Radakov, D. V. (1973). Schooling and ecology of fish. New York: Wiley and Sounds Inc.

Reebs, S. G. (2000). Can a minority of informed leaders determine the foraging movements of a fish shoal? Animal Behaviour 49, 403-409.

Rehnberg, B. G. \& Smith, R. J. F. (1988). The influence of alarm substance and shoal size on the behaviour of zebra danios, Brachydanio rerio (Cyprinidae). Journal of Fish Biology 33, 155-163. doi: 10.1111/j.1095-8649.1988.tb05457.x

Romey, W. L. (1996). Individual differences make a difference in the trajectories of simulated schools of fish. Ecological Modelling 92, 65-77.

Shaw, E. (1970). Schooling in fishes: critique and review. In: Development and evolution of behaviour (Aronson L.R. et al., eds.), pp. 452-480. San Francisco: Freeman.

Shaw, E. (1978). Schooling fishes. American Scientist 66, 166-175.

Shaw, E. \& Tucker, A. (1965). The optomotor reaction of schooling carangid fishes. Animal Behaviour 13, 330-336. 
Sogard, S. M. \& Olla, B. L. (1997). The influence of hunger and predation risk on group cohesion in a pelagic fish, walleye Pollack Theragra chalcogramma. Environmental Biology of Fishes 50, 405-413.

Viscido, S. V., Parrish, J. K. \& Grünbaum, D. (2004). Individual behavior and emergent properties of fish schools: a comparison of observation and theory. Marine Ecology Progress Series 273, 239-249.

Viscido, S.V., Parrish, J.K. \& Grünbaum, D. (2005). The effect of population size and number of influential neighbors on the emergent properties of fish schools. Ecological Modelling 183, 347-363.

Warburton, K. (1997). Social forces in animal congregations: Interactive, motivational, and sensory aspects. In Animal groups in three dimensions (Parrish, J.K. \& Hamner W.M., eds.), pp. 313-336. Cambridge: Cambridge University Press.

Warburton, K. \& Lazarus, J. (1991). Tendency-distance models of social cohesion in animal groups. Journal of Theoretical Biology 150, 473-488.

Wu, J. \& David, J. L. (2002). A spatially explicit hierarchical approach to modelling complex ecological systems: theory and applications. Ecological Modelling 153, $7-26$. 


\section{TABLES}

Table I. Mean and standard deviation of each variable by species depending on group size and statistical results. P values are the levels of significance of the differences between group sizes according to one factor ANOVAs. NND and Polarity of the bigeye scad were tested with transformed values.

\begin{tabular}{|c|c|c|c|c|c|c|c|}
\hline & \multirow{2}{*}{$\begin{array}{l}\text { Group } \\
\text { size }\end{array}$} & \multirow{2}{*}{ Small (1) } & \multirow{2}{*}{ Medium (2) } & \multirow{2}{*}{ Large (3) } & \multicolumn{3}{|c|}{$P$ value } \\
\hline & & & & & $1-2$ & $2-3$ & $1-3$ \\
\hline \multirow{5}{*}{$\begin{array}{l}\text { Bigeye } \\
\text { scad }\end{array}$} & $\mathrm{n}$ & 145 & 145 & 145 & - & - & - \\
\hline & Polarity & $0.89 \pm 0.08$ & $0.89 \pm 0.09$ & $0.81 \pm 0.12$ & NS & $\star * *$ & $\star * *$ \\
\hline & Expanse & $0.27 \pm 0.10$ & $0.28 \pm 0.10$ & $0.22 \pm 0.07$ & NS & $* * *$ & $\star * *$ \\
\hline & $\begin{array}{l}\text { Speed } \\
(\mathrm{m} / \mathrm{s})\end{array}$ & $0.47 \pm 0.17$ & $0.53 \pm 0.17$ & $0.34 \pm 0.22$ & $\star \star \star *$ & $* * \star$ & $\star \star \star *$ \\
\hline & $\begin{array}{l}\text { NND } \\
(\mathrm{m})\end{array}$ & $0.54 \pm 0.20$ & $0.24 \pm 0.12$ & $0.12 \pm 0.04$ & 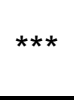 & 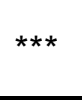 & $\star \star \star *$ \\
\hline \multirow{5}{*}{$\begin{array}{l}\text { Barred } \\
\text { flagtail }\end{array}$} & $\mathrm{n}$ & 145 & 145 & 145 & - & - & - \\
\hline & Polarity & $0.79 \pm 0.16$ & $0.51 \pm 0.19$ & $0.32 \pm 0.17$ & $\star \star \star *$ & $\star \star \star *$ & $\star \star \star ~$ \\
\hline & Expanse & $0.21 \pm 0.08$ & $0.55 \pm 0.17$ & $0.74 \pm 0.16$ & $\star \star \star *$ & $\star \star * *$ & $* \star \star$ \\
\hline & $\begin{array}{l}\text { Speed } \\
(\mathrm{m} / \mathrm{s})\end{array}$ & $0.36 \pm 0.13$ & $0.28 \pm 0.13$ & $0.16 \pm 0.12$ & $\star \star \star *$ & $\star \star \star *$ & 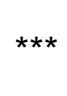 \\
\hline & $\begin{array}{l}\text { NND } \\
(\mathrm{m})\end{array}$ & $0.41 \pm 0.15$ & $0.42 \pm 0.15$ & $0.33 \pm 0.11$ & NS & $\star \star \star *$ & $\star \star \star *$ \\
\hline
\end{tabular}

***: $\mathrm{P}<0.001$; NS: not significant. 
Table II. Product-moment correlation coefficients for variable and for each species, with the significance of correlation ( $\mathrm{t}$ test). Correlations for $N N D$ (nearest neighbour distance) and Polarity of the bigeye scad were calculated on transformed values.

\begin{tabular}{llllll}
\hline Species & Variable & Polarity & Expanse & NND & Speed \\
\hline & Group size & $-0.35^{* * *}$ & $-0.24^{* * *}$ & $-0.84^{* * *}$ & $-0.35^{* * *}$ \\
Bigeye scad & Polarity & - & $-0.02^{\mathrm{NS}}$ & $0.18^{* * *}$ & $0.56^{* * *}$ \\
$(\mathrm{n}=435)$ & Expanse & - & - & $0.75^{* * *}$ & $-0.02^{\mathrm{NS}}$ \\
& NND & - & - & - & $0.17^{* * *}$ \\
\hline \multirow{2}{*}{ Barred } & Group size & $-0.71^{* * *}$ & $0.80^{* * *}$ & $-0.26^{* * *}$ & $-0.56^{* * *}$ \\
flagtail & Polarity & - & $-0.70^{* * *}$ & $0.02^{\mathrm{NS}}$ & $0.64^{* * *}$ \\
$(\mathrm{n}=435)$ & Expanse & - & - & $0.09^{\mathrm{NS}}$ & $-0.42^{* * *}$ \\
& NND & - & - & - & $0.19^{* * *}$ \\
\hline
\end{tabular}

$* * \mathrm{P}<0.01 ; * * * \mathrm{P}<0.001 ; \mathrm{NS}$ : not significant. 
Table III. Results of the MANOVA (adjusted R-square $=0.34$ ) on the Polarity of the bigeye scad fishes $(n=435)$.

\begin{tabular}{lllll}
\hline & Sum of Square & d.f. & $\mathrm{F}$ & $\mathrm{p}$ \\
\hline Intercept & 0.0420 & 1 & 77.22 & 0.0001 \\
Speed & 0.0632 & 1 & 116.08 & 0.0001 \\
Group Size & 0.0081 & 2 & 7.41 & 0.0007 \\
NND & 0.0014 & 1 & 2.48 & 0.1158 \\
Expanse & 0.0001 & 1 & 0.16 & 0.6881 \\
Error & 0.2330 & 428 & & \\
\hline
\end{tabular}




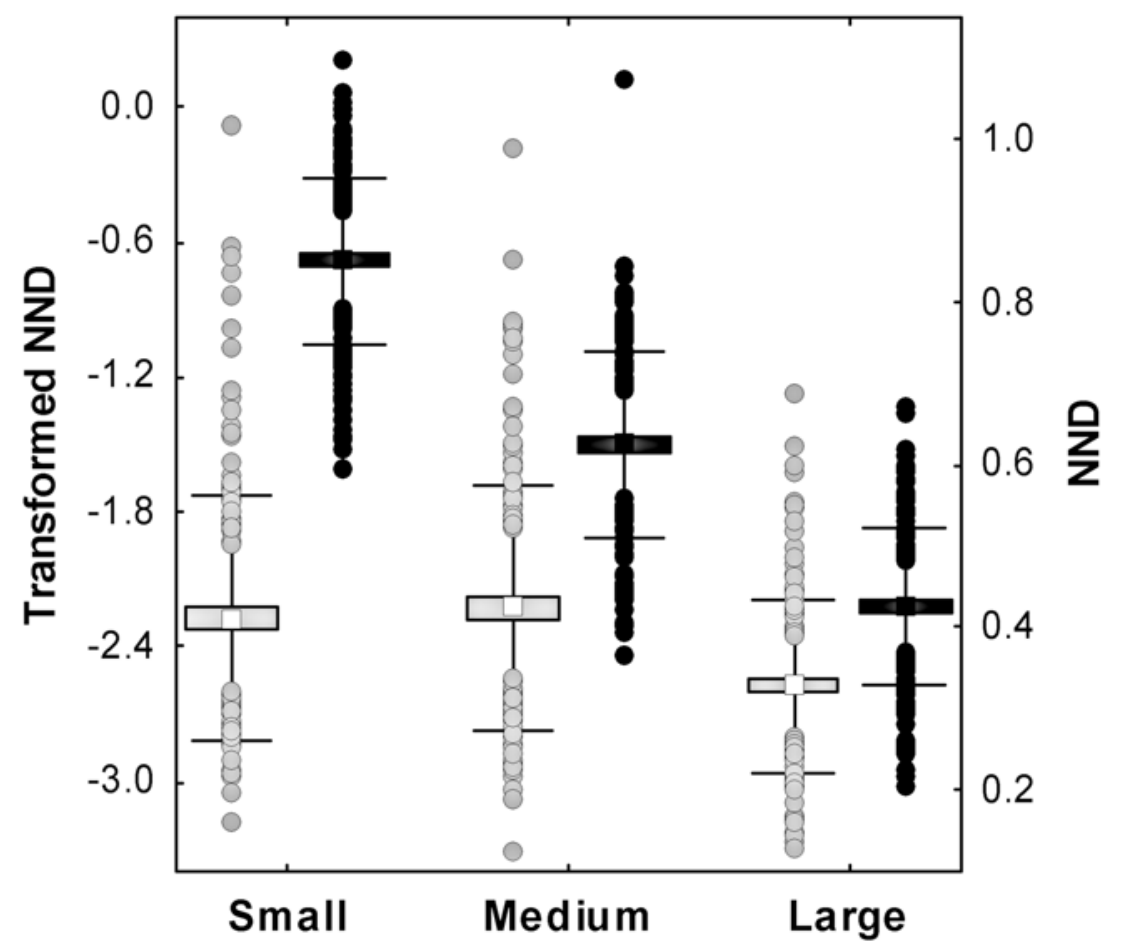

Figure 1. Categorized box plot of the nearest neighbour distance $(N N D) v s$. the group size for the bigeye scad. ( $\mathbf{\square}$ : Mean, $\square: \pm$ SE,工 : $\pm 0.95 * \mathrm{SD}, \bullet$ : Extreme) and the barred flagtail $\square$ : Mean, $\square: \pm$ SE, $工: \pm 0.95 * \mathrm{SD}, 0$ : Extreme). $N N D$ of the bigeye scad were calculated on transformed values. 


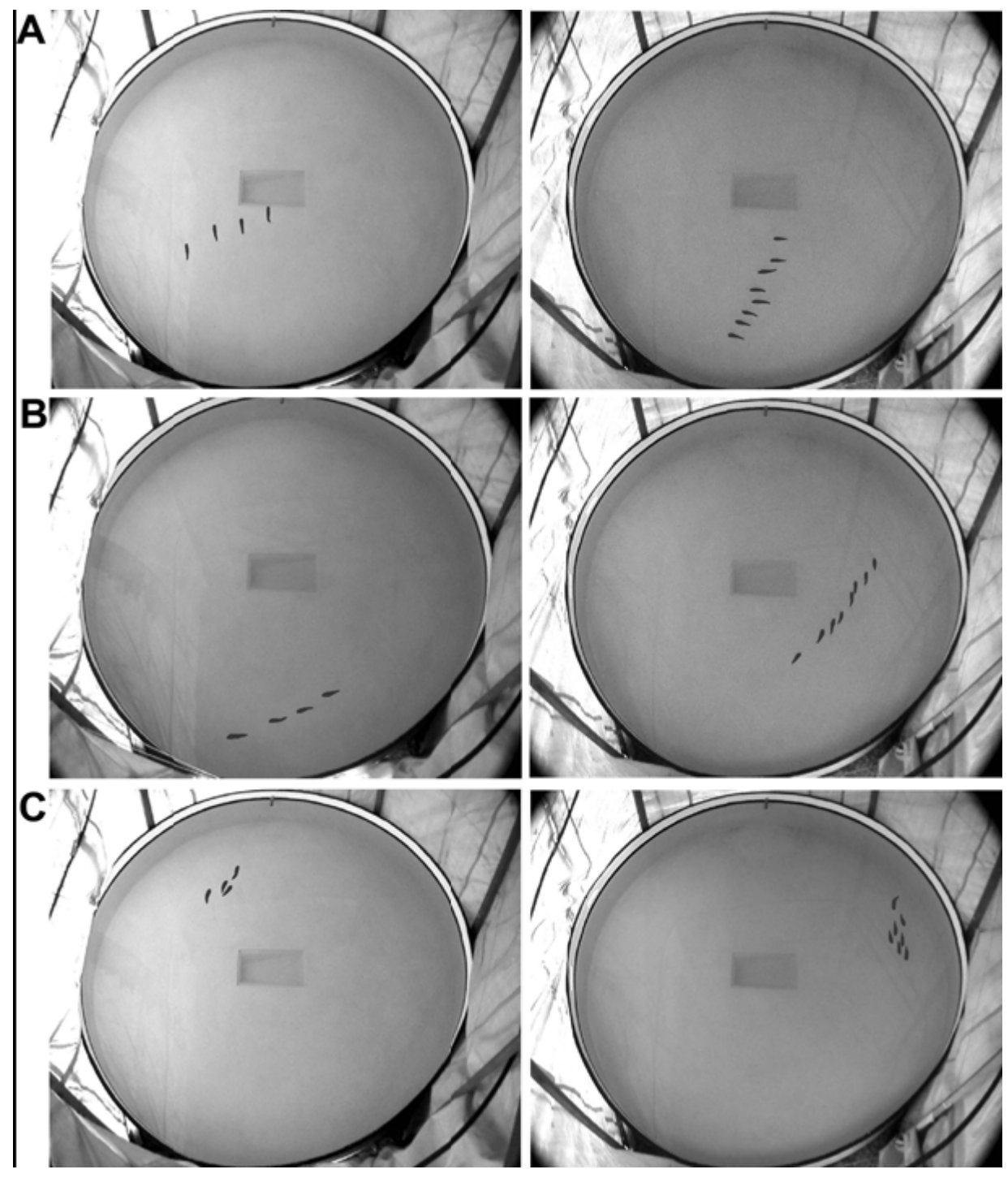

Figure 2. Images resulting from the observations carried out on groups of four (left) and eight (right) bigeye scads, illustrating the three patterns displayed by the fish (A: line, B: column, C: dense school). 